\title{
Aberrações ópticas de alta ordem em pacientes com distonias faciais tratados com toxina botulínica
}

\author{
Ocular wavefront aberrations in patients with facial dystonia treated with botulinum toxin
}

Mariann Midori Yabiku ${ }^{1}$, Juliana de Fillipl Sartori ${ }^{1}$, Eduardo Pantaleão Sarraff ${ }^{1}$, Tammy Hentona Osakı ${ }^{2}$, Sidarta Keizo Hossaka ${ }^{2,3}$, Carolina Isolane Pereira ${ }^{2,3}$, Wilson de Freitas ${ }^{3}$, Midori Hentona Osaki ${ }^{2}$, Angelino Julio Cariello ${ }^{2}$

\section{RESUMO}

Objetivo: Avaliar as aberrações ópticas de alta ordem em pacientes com distonias faciais tratados com toxina botulínica tipo A.

Métodos: Pacientes com diagnóstico clínico de espasmo hemifacial ou blefaroespasmo essencial em atividade foram submetidos ao exame biomicroscópico e à análise de frente de ondas através do aberrômetro Alcon LADARvision ${ }^{\circledR}$, sob midríase medicamentosa. A seguir, foram tratados com injeções de toxina botulínica tipo A. Após um mês, a análise de frente de ondas foi repetida da mesma forma e pelo mesmo oftalmologista. As aberrações de alta ordem foram comparadas antes e após o tratamento. O teste T pareado foi utilizado para comparar os valores numéricos antes e após o tratamento.

Resultados: Foram incluídos no estudo um total de 11 pacientes, 6 com blefaroespasmo essencial (54,5\%) e 5 com espasmo hemifacial (45,5\%). Nos pacientes com espasmo hemifacial foram analisados apenas o lado acometido, totalizando 17 olhos com espasmo. A idade variou de 50 a 72 anos, com média de $65,9 \pm 8,2$ anos. Oito pacientes eram do sexo feminino (72,7\%), sendo a relação masculino/feminino de 1:2,6. A média do "root mean square" (RMS) das aberrações de alta ordem foi 0,68 antes e 0,63 após um mês do tratamento $(p=0,01)$. A média da aberração esférica foi de 0,23 e 0,17 antes e após o tratamento respectivamente $(p=0,01)$. Não houve diferenças estatisticamente significantes nos demais tipos de aberrações de alta ordem após o tratamento $(p>0,05)$

Conclusão: $\mathrm{O}$ tratamento com toxina botulínica $\mathrm{A}$ pode diminuir as aberrações esféricas em pacientes com distonias faciais.

Descritores: Blefaroespasmo/quimioterapia; Espasmo hemifacial/quimioterapia; Toxinas botulínicas tipo A/uso terapêutico; Distonia; Músculos faciais/fisiopatologia; Astigmatismo/quimioterapia; Indice de gravidade de doença

\begin{abstract}
Purpose: To analyze the ocular wavefront aberrations in patients with facial dystonia treated with botulinum toxin $A$.

Methods: Patients with benignessential blepharospasm and hemifacial spasm in activity underwent slit lamp examination and bilateral wavefront analysis under pharmacologic mydriasis using Alcon LADARvision ${ }^{\circledR}$ wavefront aberrometry system. After that, all patients were treated with botulinum toxin A injections performed by the same ophthalmologist. After one month, the wavefront analysis was performed in the same way and by the same examiner. The main outcome measure was the change in ocular wavefront aberrations. Paired T-test was used to compare pre and post-injection numeric wavefront values.

Results: From a total of 11 patients enrolled in this study, 6 (54.5\%) had essential blepharospasm and 5 (45.5\%) had hemifacial spasm. The fellow eyes of patients with hemifacial spasm were not included, totalizing 17 eyes with spasm. Eight patients were female $(72.7 \%)$ and three were male $(27.3 \%)$, the male:female ratio was 1:2.6. The age ranged from 50 to 72 years old with a mean of $65.9 \pm 8.2$ years. The mean of high order root mean square (RMS) wavefront aberrations was 0.68 before and 0.63 one month after the treatment $(p=0.01)$. Before the treatment, the mean of spherical aberration was 0.23 and decreased to 0.17 one month after the treatment $(p=0.01)$. There was no significant difference in the other higher-order aberrations before and after the treatment ( $p>0.05)$.
\end{abstract}

Conclusion: The treatment with botulium toxin may decrease spherical aberrations in patients with facial dystonia.

Keywords: Blepharospasm/drug therapy; Hemifacial spasm/drug therapy; Botulinum toxins, type A/therapeutic use; Dystonia; Facial muscles/physiopathology; Astigmatism/ drug therapy; Severity of illness index

\section{INTRODUÇÃO}

Blefaroespasmo essencial (BE) é uma doença caracterizada por contrações involuntárias, espasmódicas, bilaterais e simétricas dos músculos perioculares (orbicularis oculi, procerus e corrugador) ${ }^{(1,2)}$. Apresenta etiologia incerta e geralmente inicia-se entre $5^{\underline{a}}$ a $6^{\underline{a}}$ década de vida, acometendo mais mulheres ${ }^{(3,4)}$. Pode causar cegueira funcional pelo persistente fechamento palpebral, levando a importante limitação das atividades sociais ${ }^{(5)}$.

Espasmo hemifacial (EH) é caracterizado por contrações tônicoclônicas unilaterais e involuntárias dos músculos inervados pelo nervo facial ipsilateral, respeitando a linha média, podendo, menos frequentemente, ser bilateral e, neste caso, as contrações são assimétricas ${ }^{(1,2)}$. Embora alguns pacientes possam ser geneticamente predispostos a desenvolver EH, a maioria dos casos são esporádicos(6). Estudos apontam como etiologia a compressão do nervo facial por lesões vasculares próximo a sua emergência no tronco cerebral. Dessa forma, uma minuciosa investigação neurológica é necessária ${ }^{(1,5)}$.

A toxina botulínica tipo A, considerada tratamento de eleição, bloqueia a liberação de acetilcolina na junção neuromuscular e causa paralisia muscular temporária diminuindo consideravelmente as contrações palpebrais e melhorando a qualidade de vida dos pacientes ${ }^{(4,5)}$.
Submitted for publication: May 2, 2011

Accepted for publication: October 25, 2011

Study carried out at the Departamento de Oftalmologia, Universidade Federal de São Paulo UNIFESP - São Paulo, SP, Brazil.

Physician, Departamento de Oftalmologia, Universidade Federal de São Paulo - UNIFESP - São Paulo (SP), Brazil.

2 Physician, Setor de Cirurgia Plástica Ocular, Departamento de Oftalmologia, Universidade Federal de São Paulo - UNIFESP - São Paulo (SP), Brazil.

Physician, Setor de Cirurgia Refrativa, Departamento de Oftalmologia, Universidade Federal de São Paulo - UNIFESP - São Paulo (SP), Brazil.
Funding: No specific financial support was available for this study.

Disclosure of potential conflicts of interest: M.M.Yabiku, None; J.F.Sartori, None; E.P.Sarraff, None; T.H.Osaki, None; S.K.Hossaka, None; C.I.Pereira, None; W.de Freitas, None; M.H.Osaki, None; A.J.Carriello, None.

Correspondence address: Angelino Julio Cariello. Hospital de Olhos Sadalla Amin Ghanem. Rua Abdon Batista, 146 - Joinville (SC) - 89201-010 - Brazil - E-mail: angelino65@yahoo.com

Este trabalho foi avaliado e aceito pelo comitê de ética em pesquisa da Universidade Federal de São Paulo (UNIFESP) sob número: 1625/09 
Diversos estudos demonstraram que a pressão das pálpebras tem um efeito direto no formato da córnea e na indução de astigmatismo. Crianças frequentemente apresentam astigmatismos a favor da regra que tendem a se modificar no sentido contra a regra com a diminuição da tensão palpebral induzida pela senilidade ${ }^{(7)}$. Modificações no astigmatismo também foram documentadas após cirurgias de blefaroptose e dermatocalase superior ${ }^{(8-10)}$ e, além disso, várias condições que acometem as pálpebras superiores ou inferiores, incluindo calázio ${ }^{(11)}$, implantes de peso de ouro ${ }^{(12)}$ e hemangiomas ${ }^{(13)}$ podem afetar o formato da córnea.

Estudo envolvendo pacientes com distonias faciais encontrou maiores astigmatismos nos olhos afetados de pacientes com EH comparados com olho contralateral e verificou que o tratamento com toxina botulínica altera a curvatura da córnea, diminuindo astigmatismos a favor da regra e aumentando astigmatismo contra a regra ${ }^{(7)}$.

O objetivo do presente estudo foi analisar o efeito do tratamento com toxina botulínica nas aberrações ópticas de alta ordem em pacientes com distonias faciais.

\section{MÉTODOS}

Este estudo foi aprovado pelo comitê de ética em pesquisa da instituição e seguiu as diretrizes da Declaração de Helsinque de 1964, suas versões posteriores e da resolução 196/96 do Conselho Nacional de Saúde/Ministério da Saúde para pesquisa em seres humanos.

Pacientes com diagnóstico clínico de BE ou EH, oriundos do Departamento de Oftalmologia da Universidade Federal de São Paulo, foram convidados a participar do estudo por meio de assinatura de termo de consentimento livre e esclarecido. Alergia conhecida à toxina botulínica ou componente da medicação, presença de qualquer outra doença sistêmica ou ocular prévia que poderiam interferir com os resultados do estudo e tratamento prévio com toxina botulínica há menos de seis meses foram considerados critérios de exclusão.

Todos os pacientes foram submetidos ao exame clínico ectoscópico e biomicroscopia em lâmpada de fenda e, em seguida, submetidos a dilatação pupilar com tropicamida 1\%. A análise de frente de ondas foi realizada por um único oftalmologista, através do aberrômetro LADARvision ${ }^{\circledR}$ (Alcon, Fort Worth, Texas, EUA) conforme orientações do fabricante.

Na sequência, todos os pacientes foram tratados com toxina botulínica A (Botox ${ }^{\circledR}$, Allergan, Irvine, Califórnia, EUA) pelo mesmo médico conforme protocolo de aplicação clínica padronizada, diluindo-se a toxina imediatamente antes da aplicação em solução de cloreto de sódio estéril 0,9\% para uma concentração final de 50 unidades $/ \mathrm{ml}$. Volume de $0,05 \mathrm{ml}(2,5 \mathrm{U})$ por ponto de aplicação foi injetado por via intramuscular nos músculos orbicular do olho, próceros e corrugadores dos supercílios bilateralmente em casos de BE e, em casos de EH, também aplicado nos músculos zigomáticos e risório conforme ilustra a figura $1^{(14)}$. Após 30 dias da aplicação da toxina, a análise de frente de ondas foi repetida da mesma forma, pelo mesmo examinador do início do estudo.

Por meio do programa SPSS ${ }^{\circledR}$ (Windows, versão 16), foi empregado o teste $T$ pareado para comparar as médias dos valores numéricos da aberrometria antes e depois da aplicação de toxina botulínica.

\section{RESULTADOS}

Foram incluídos no estudo 11 pacientes, sendo 6 com BE (54,5\%) e $5 \mathrm{com}$ EH (45,5\%). Nos pacientes com EH foram analisados apenas o lado acometido, totalizando 17 olhos com espasmo. A idade dos pacientes variou de 50 a 72 anos, com média de 65,9 \pm 8,2 anos. Oito pacientes eram do sexo feminino $(72,7 \%)$, sendo a relação masculino/feminino de 1:2,6. A média do "root mean square" (RMS) foi 0,68 antes e 0,63 após um mês do tratamento $(p=0,01)$. Antes do tratamento, a média da aberração esférica foi 0,23 e diminuiu para 0,17 um mês após o tratamento $(p=0,01)$. Não houve diferenças estatisticamente significantes nos demais tipos de aberrações de alta ordem após o tratamento $(p>0,05)$. Antes do tratamento, as médias das aberrações de baixa ordem (defocus, astigmatismo e coma) foram, respectivamente, 2,7; 0,8 e 0,4. Após um mês do tratamento, esses valores foram respectivamente, 2,6; 0,8 e 0,45 $(p>0,05)$ - tabela 1.

Todos os pacientes apresentaram melhora sintomática, diminuição do padrão de contrações e nenhum efeito adverso ou complicação foi identificada.

\section{DISCUSSÃO}

Embora estudos prévios já descrevessem mudanças topográficas induzidas pelo piscar ou por pressão exercida pelas pálpebras sobre a córnea ${ }^{(7-13)}$, o presente estudo foi pioneiro em avaliar as aberrações de alta ordem em pacientes com distonias faciais antes e após tratamento com toxina botulínica.

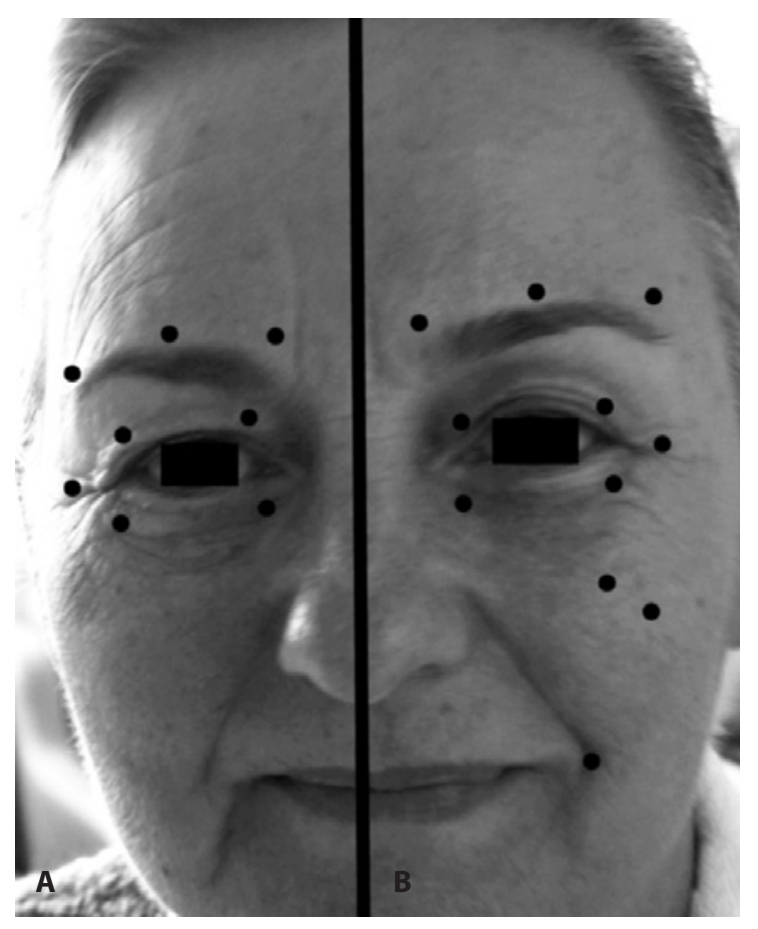

$A=$ blefaroespasmo essencial; $B=$ espasmo hemifacial

Figura 1. Pontos de aplicação.

Tabela 1. Comparação das médias dos valores de aberrações ópticas de pacientes com distonias faciais antes e um mểs após o tratamento com toxina botulínica

\begin{tabular}{lccc}
\hline & $\begin{array}{c}\text { Antes do } \\
\text { tratamento }\end{array}$ & $\begin{array}{c}\text { Um mês após } \\
\text { o tratamento }\end{array}$ & $\begin{array}{c}\text { Significância } \\
\text { estatística }\end{array}$ \\
\hline RMS & 0,68 & 0,63 & $\mathrm{p}=0,011^{*}$ \\
Aberração esférica & 0,23 & 0,17 & $\mathrm{p}=0,014^{*}$ \\
Defocus & 2,66 & 2,62 & $\mathrm{p}=0,260$ \\
Astigmatismo & 0,80 & 0,77 & $\mathrm{p}=0,316$ \\
Coma & 0,44 & 0,45 & $\mathrm{p}=0,400$ \\
\hline
\end{tabular}

RMS= root mean square

*=estatisticamente significante (teste T para amostras pareadas) 
O aberrômetro LADARvision ${ }^{\circledR}$ utiliza o sistema Shack-Hartmann, que se baseia na análise da frente de onda que emerge do olho. 0 seu sensor utiliza um feixe de laser (diodo) que é direcionado para a mácula, sendo então refletido de volta, passando pelo vítreo, cristalino, pupila, câmara anterior, córnea e filme lacrimal. Qualquer aberração óptica criada por essas estruturas irá determinar uma modificação específica no feixe de luz. Após a saída do olho, esse raio de luz atravessa um sistema de lentes que concentram a frente de onda, que será representada por uma grade de pontos. Essas informações são capturadas por um sistema computadorizado que compara as imagens oriundas do olho com uma frente de onda plana, livre de aberrações. As diferenças entre o feixe capturado e a frente de onda plana representarão as aberrações do olho(15).

O presente estudo demonstrou diminuição estatisticamente significante da média do "root mean square" (RMS), que mede a quantidade total de aberrações de um sistema óptico (15), sugerindo genericamente uma diminuição das aberrações de alta ordem com o tratamento com toxina botulínica. A análise das medidas qualitativas específicas das aberrações encontradas identificou diminuição da aberração esférica após um mês de tratamento.

A aberração esférica em um sistema óptico faz com que os raios luminosos sejam refratados mais intensamente quanto mais distantes do centro óptico do sistema, podendo clinicamente causar sintomas como halos e borramento de pontos luminosos (starburst) principalmente à noite devido à dilatação pupilar escotópica que permite maior entrada de raios periféricos refratados ${ }^{(16)}$. Os dados do presente estudo sugerem que o tratamento com toxina botulínica poderia também proporcionar melhor qualidade de visão aos pacientes com distonias faciais, principalmente em condições de baixa luminosidade.

Embora se acredite que a ação de relaxamento da musculatura palpebral induzida pelo tratamento com toxina botulínica pode ser responsável por modificações no formato da córnea, as modificações nas aberrações identificadas no presente estudo podem também ser justificadas por possíveis variações na distribuição e composição do filme lacrimal. Estudo recente demonstrou que o tratamento de paciente com distonias faciais por injeção de toxina botulínica pode aumentar a estabilidade e melhorar a drenagem da lágrima ${ }^{(17)}$. A acomodação poderia também influenciar modificações de frente de onda(18), porém a faixa etária da amostra estudada (média de 65,9 anos) permitindo inferir que a interferência da acomodação seja nula ou mínima.

Moon et al., analisaram mudanças topográficas de pacientes com BE e EH e verificaram diminuição do astigmatismo a favor da regra e aumento do astigmatismo contra a regra após 1 mês de tratamento com toxina botulínica, sugerindo que as pálpebras podem desempenhar um importante papel no estado refracional do olho ${ }^{(7)}$. Na presente amostra estudada não foi observada diferença significante na média das demais aberrações de alta ordem ou nas aberrações de baixa ordem (defocus, astigmatismo e coma) após um mês de tratamento. A raridade da doença, somada aos critérios de exclusão que pudessem falsear os resultados como doenças de superfície ocular externa, opacidade de meios, tratamento prévio com toxina botulínica há menos de seis meses, entre outros já citados, limitou o tamanho da nossa amostra, o que pode ter sido responsável pela ausência de mudança significativa das demais aberrações.

\section{CONCLUSÃO}

O tratamento com toxina botulínica A pode diminuir as aberrações esféricas em pacientes com distonias faciais e possivelmente melhorar a qualidade de visão escotópica.

\section{REFERÊNCIAS}

1. Kenney C, Jankovic J. Botulinum toxin in the treatment of blepharospasm and hemifacial spasm. J Neural Transm. 2008:115(4):585-91.

2. Carvalho RM, Gomi CF, Carvalho AL, Matayoshi S, Moura EM. Tratamento do blefaroespasmo e distonias faciais correlatas com toxina botulínica - estudo de 16 casos. Arq Bras Oftalmol. 2003;66(1):13-6

3. Schellini AS, Matai O, Igami TZ, Padovani R, Pavodani CP. Blefaroespasmo essencial e espasmo hemifacial; características dos pacientes, tratamento com toxina botulínica Ae revisão da literatura. Arq Bras Oftalmol. 2006;69(1):23-6.

4. Defazio G, Livrea P. Primary blepharospasm: diagnosis management. Drugs. 2004; 64(3):237-44.

5. Osaki MH, Belfort R. Qualidade de vida e custos diretos em pacientes com blefaroespasmo essencial e espasmo hemifacial, tratados com toxina botulínica-A. Arq Bras Oftalmol. 2004;67(1):43-9.

6. Micheli F, Scorticati MC, Gatto E, Cersosimo G, Adi J. Familial hemifacial spasm. Mov Disord. 1994:9(3):330-2

7. Moon NJ, Lee HI, Kim JC. The changes in corneal astigmatism after botulinum toxin-A injection in patients with blepharospasm. J Korean Med Sci. 2006;21(1):131-5.

8. Zinkernagel MS, Ebneter A, Ammann-Rauch D. Effect of upper eyelid surgery on corneal topography. Arch Ophthalmol. 2007;125(12):1610-2.

9. Shao W, Byrne P, Harrison A, Nelson E, Higler P. Persistent blurred vision after blepharoplasty and ptosis repair. Arch Facial Plast Surg. 2004;6(3):155-7.

10. Brown MS, Siegel IM, Lisman RD. Prospective analysis of changes in corneal topography after upper eyelid surgery. Ophthal Plast Reconstr Surg. 1999;15(6):378-83.

11. Bagheri A, Hasani HR, Karimian F, Abrishami M, Yazdani S. Effect of chalazion excision on refractive error and corneal topography. Eur J Ophthalmol. 2009;19(4):521-6.

12. Mavrikakis I, Beckingsale $P$, Lee E, Riaz Y, Brittain P. Changes in corneal topography with upper eyelid gold weight implants. Ophthal Plast Reconstr Surg. 2006;22(5):331-4.

13. Robb RM. Refractive errors associated with hemangiomas or the eyelids and orbit in infancy. Am J Ophthalmol. 1977;83(1):52-8

14. Ortisi E, Henderson HW, Bunce C, W Xing, Collin JR. Blepharospasm and hemifacial spasm: a protocol for titration of botulinum toxin dose to the individual patient and for the management of refractory cases. Eye (Lond).2006;20(8):916-22.

15. Vieira Netto M, Ambrósio Junior R, Schor P, Chalita MR, Chamon W, editores. Wavefront, topografia e tomografia da córnea e segmento anterior: atualização propedêutica em cirurgia refrativa. Rio de Janeiro: Cultura Médica; 2006. p.253.

16. Chalita MR, Chavala S, Xu M, Krueger RR. Wavefront analysis in post-LASIK eyes and its correlation with visual symptoms, refraction, and topography. Ophthalmology. 2004; 111(3):447-53

17. Oliveira FC, Oliveira GC, Cariello AJ, Felberg S, Osaki MH. Influência da toxina botulínica tipo A na função lacrimal em pacientes com distonias faciais. Arq Bras Oftalmol. 2010; 73(5):405-8.

18. Tarrant J, Roorda A, Wildsoet CF. Determining the accommodative response from wavefront aberrations. J Vis. 2010;10(5):4 